\title{
Interleukin-25 is detrimental for recovery after spinal cord injury in mice
}

Dearbhaile Dooley ${ }^{1,2}$, Evi Lemmens $^{1}$, Peter Ponsaerts ${ }^{2}$ and Sven Hendrix ${ }^{1 *}$

\begin{abstract}
Background: The cytokine, interleukin (IL)-25, is thought to be critically involved in inducing a type 2 immune response which may contribute to regeneration after central nervous system (CNS) trauma. We investigated whether applying recombinant IL-25, locally or systemically, in a mouse model of spinal cord injury (SCI) improves functional and histological recovery.

Findings: Repeated systemic administration of IL-25 did not influence functional recovery following SCI. In contrast, a single local administration of IL-25 significantly worsened locomotor outcome, which was evident from a decreased Basso mouse scale (BMS) score compared with phosphate-buffered saline (PBS)-treated controls. This was accompanied by a significant increase in lesion size, demyelination, and T helper cell infiltration.

Conclusions: These data show for the first time that IL-25 is either ineffective when applied systemically or detrimental to spinal cord recovery when applied locally. Our findings question the potential neuroprotective role of IL-25 following CNS trauma.
\end{abstract}

Keywords: IL-25, Type 2 immune response, Spinal cord injury, Basso mouse scale, Locomotor recovery, Th1, Th2

\section{Findings Introduction}

For decades, it has been the general opinion that an imbalanced immune response plays a major role in the pathophysiology of central nervous system (CNS) trauma and disease. Inflammation may prove problematic for many repair processes [1] but may also exert beneficial effects when closely regulated. The type 2 response can be characterized by differentiation of $\mathrm{CD} 4^{+}$ $\mathrm{T}$ helper type 2 (Th2) cells and the production of the type 2 cytokines interleukin-4 (IL-4), IL-5, IL-9, and IL-13 [2-4]. This in turn inhibits phagocytosis [5] and suppresses inflammatory cytokines [6]. Therefore, type 2 immune factors can contribute to immune regulation by suppressing excessive pro-inflammatory processes $[7,8]$.

We as well as others have shown that cytokines associated with Th2 cells such as IL-4 [9-11] and IL-10 $[12,13]$, not only promote neuronal survival and regeneration but also improve functional outcome after CNS

\footnotetext{
* Correspondence: sven.hendrix@uhasselt.be

'Department of Morphology, Biomedical Research Institute, Hasselt

University, Diepenbeek, Belgium

Full list of author information is available at the end of the article
}

trauma such as SCI. IL-25 (also known as IL-17E) has been suggested to be a key player in the origin of a type 2 response $[2,14]$. While research has begun to unravel its importance in immunity in general, conclusive data on the role of IL-25 in the CNS is lacking. Although a limited number of studies are currently available, these tend to point towards a protective role of IL-25 in neuroinflammation (reviewed in $[15,16]$ ). For example, IL-25 treatment suppresses Th17 responses and disease symptoms in experimental autoimmune encephalomyelitis (EAE) and is important in maintaining bloodbrain barrier function [17, 18]. IL-25 expression is downregulated by pro-inflammatory cytokines such as tumor necrosis factor- $\alpha$ and IL-1 $\beta$, which increase acutely after trauma. Consistently, IL-25 is reduced in the pro-inflammatory milieu of CNS lesions [17]. These findings suggest that an increase in IL-25 may possess the therapeutic potential to provide repair after CNS trauma.

In the present short report, we tested whether recombinant murine IL-25, administered either as a single dose locally to the spinal cord or via repeated systemic injections, improves functional recovery after SCI in mice. While no clinical effect was observed following 
systemic administration of IL-25, surprisingly, when applied locally, IL-25 lead to a significant decrease in locomotor recovery as well as a substantial increase in lesion size, demyelination, and $\mathrm{T}$ helper cell infiltration.

\section{Methods}

A T-cut hemisection injury was performed as previously described [19-22] in 10-week-old female BALB/c mice (Harlan, The Netherlands). See supplementary materials for details. Mice were treated with recombinant murine IL-25 (500 ng or $1 \mu \mathrm{g}$; ImmunoTools, Germany) via two different methods. Mice received either a single, local application of IL-25 (1 $\mu \mathrm{g})$, by placing a cytokinesaturated gelfoam patch at the lesion site immediately after injury, or systemic administration via repeated intraperitoneal (i.p.) injections (500 ng) once daily for 7 days starting 1 day before injury. The dose for the local application of IL-25 was chosen based on pilot experiments in our lab, where we observed a non-significant trend towards a decreased functional recovery after SCI following treatment with a lower dose $(500 \mathrm{ng} / \mathrm{ml}$; data not shown). The dose for systemic administration was chosen based on a previous study [17]. Control animals were treated with vehicle, i.e., phosphate-buffered saline (PBS) ( $n=7-10$ mice/group). All experiments were performed according to the guidelines of EU Directive 2010/63/EU on the protection of animals used for scientific purposes and were approved by the local ethical committee for animal experimentation at Hasselt University.

Starting 1 day after surgery, functional recovery in SCI mice was measured at regular time points for 3 weeks using the Basso mouse scale (BMS) [23] as previously described [19, 20, 22]. Histological analysis was performed on mice receiving a local and systemic application of IL-25 as previously described [20, 22]. The following antibodies were analyzed: anti-glial fibrillary acidic protein (GFAP; Sigma-Aldrich, Belgium), antimyelin basic protein (MBP; Millipore, Belgium), antiCD4 (BD biosciences, Belgium), and anti-ionized calcium-binding adaptor molecule 1 (Iba-1; Wako, Germany). See Additional file 1 for details.

For quantification of astrogliosis (GFAP) and microglia/ macrophage infiltration (Iba-1), TissueQuest immunofluorescence analysis software (TissueGnostics $\mathrm{GmbH}$, v3.0) was used, as previously described [24]. Pictures were taken using a Nikon Eclipse 80i microscope (Nikon, Brussels, Belgium). See Additional file 1 for details.

To study the effect of IL-25 on cell survival in vitro, we used a human astrocytoma cell line (CCF) [25], a human glial (oligodendrocytic) hybrid cell line (MO3.13) [26], an immortalized murine BV-2 cell line [27], and primary cortical neuronal cells as previously described [3]. All cell types were grown under optimal conditions and treated with selected concentrations of IL-25 (5 ng/ $\mathrm{ml}, 50 \mathrm{ng} / \mathrm{ml}, 500 \mathrm{ng} / \mathrm{ml}$, and $1 \mu \mathrm{g} / \mathrm{ml}$ ) for $72 \mathrm{~h}$ to measure viability. See Additional file 1 for details.

Statistical analyses were performed using GraphPad Prism software (GraphPad Software Inc., USA). Differences between treatment groups in lesion size, demyelinated area, and $\mathrm{T}$ cell numbers were calculated using the Mann-Whitney $U$ test. Differences in astrogliosis and microglia/macrophage infiltration, as well as in the BMS data, were analyzed using the two-way ANOVA for repeated measurements (with Bonferroni post hoc tests). Differences were considered to be significant when $p<$ 0.05 . Data in graphs are presented as mean \pm SEM.

\section{Results and discussion}

In this short report, we investigated whether increasing levels of IL-25, a potential inducer of a type 2 immune response, can promote functional recovery in a mouse model of SCI. Considering the widespread expression of the receptor A subunit of the IL-17 receptor which forms a complex with the receptor B subunit upon binding with IL-25 [28], we aimed to distinguish between local and systemic effects of treatment. We found that local application of IL-25 led to a significant worsening in motor performance following injury compared with PBS controls (Fig. 1a; " $p<0.05$ ). At the histological level, these results were accompanied by a $30 \%$ increase in lesion size (Fig. 1b, $g, \mathrm{~h} ;{ }^{* * * *} p<0.001$ ) and demyelinated area (Fig. 1c, i, j; $* *<<0.01$ ). Surprisingly, systemic IL-25 treatment did not influence functional recovery (Fig. 2a). Furthermore, there was no effect of systemic IL-25 treatment on lesion size or demyelinated area (Fig. 2b, c).

We also analyzed the presence of microglia/macrophages (Figs. 1d, k, l and 2d) as well as astrogliosis (Figs. 1e, g, h and 2e), by quantifying the number of Iba$1+$ and GFAP+ cells, respectively. However, no significant differences were found between IL-25 treated and control groups, in both local and systemic treatment. Following quantification of perilesional $\mathrm{CD} 4^{+} \mathrm{T}$ cells, we found a significant increase in the number of cells in tissue sections from mice treated locally with IL-25, compared with PBS controls (Fig. 1f, m, n; ${ }^{* * * *} p<0.001$ ). There was no effect of systemic IL-25 treatment on the number of $\mathrm{CD}_{4}^{+} \mathrm{T}$ cells (Fig. 2f). The precise role of $\mathrm{T}$ cells following CNS injury is still subject to discussion. Although they may display beneficial effects under certain conditions, accumulation of endogenous $\mathrm{T}$ cells may be considered detrimental $[7,20]$.

We also investigated the effect of IL-25 in vitro on cell viability. However, we observed no significant effects of various concentrations of IL-25 on survival of oligodendrocytes, astrocytes, microglia, or primary cortical neuronal cells (Additional file 2: Figure S1A-D). These results may be consistent with the lacking effect of 

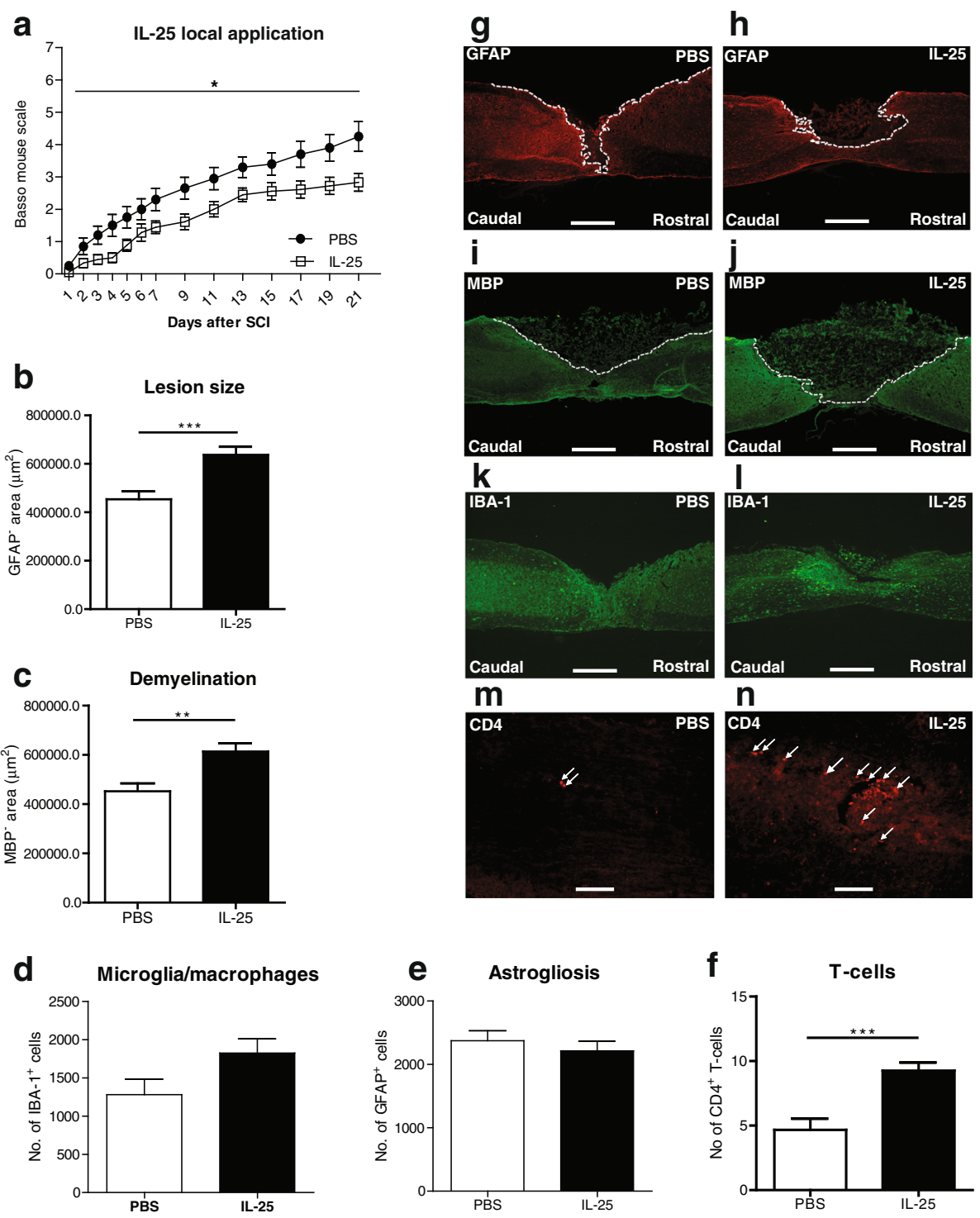

Fig. 1 Local application of IL-25 decreases functional outcome and increases lesion size, demyelination, and T cell infiltration following SCI in mice. (a) Mice receiving local application of IL-25 show a statistically significant decrease in functional outcome when compared to those receiving PBS, as measured by the BMS $\left({ }^{*} p<0.05\right), n=9-10$ mice/group. (b) Lesion size and (c) demyelinated area were quantified by staining for ( $\mathbf{g}$, $\mathbf{h}$ ) GFAP and $(\mathbf{i}, \mathbf{j})$ MBP, respectively, as depicted by the dotted white line. Image analysis revealed a significant increase in (b) lesion size and (c) demyelinated area in animals treated locally with IL-25, compared with the PBS control group. Quantification of (d) Iba- $\mathbf{1}^{+}$and (e) GFAP ${ }^{+}$cells after SCl using TissueQuest software revealed no significant difference in $(\mathbf{k}, \mathbf{l})$ microglia/macrophages numbers or $(\mathbf{g}, \mathbf{h}$ astrogliosis between animals receiving PBS or IL-25. (f) Significantly more CD4 ${ }^{+} T$ cells are present in the spinal cord sections of the (n) IL-25-treated mice, compared with ( $\left.\mathbf{m}\right)$ PBS-treated mice, 3 weeks after SCl. Scale bars of representative photomicrographs: $(\mathbf{g}-\mathbf{I})=500 \mu \mathrm{m}, \mathbf{m}+\mathbf{n}=50 \mu \mathrm{m}$. Data represent mean \pm SEM. ${ }^{* *} p<0.001$, ${ }^{* *} p<0.01$, $n=5-6$ mice/group

systemic IL-25 treatment in vivo. They also indicate that the toxic effect observed locally is not caused by a direct effect on the above cell types. This suggests that local administration of IL-25 following SCI activates an indirectly mediated cascade of detrimental immune events.

Although a member of the rather pro-inflammatory IL-17 family, IL-25 plays a somewhat different role in the context of CNS inflammation. IL-25 messenger RNA (mRNA) is highly expressed in polarized Th2 cells [14], and IL-25 administration in mice drives the Th2 response, by elevating IL-4 and IL-13 levels $[14,16]$. Systemic IL-25 regulates the development of autoimmune inflammation mediated by IL-17-producing cells and suppresses EAE symptoms in a relapse-remitting model [14]. Additionally, the delivery of IL-25 to the CNS in two different models of neuroinflammation was able to drive microglia and macrophages to a more antiinflammatory and tissue-protective phenotype [29]. 


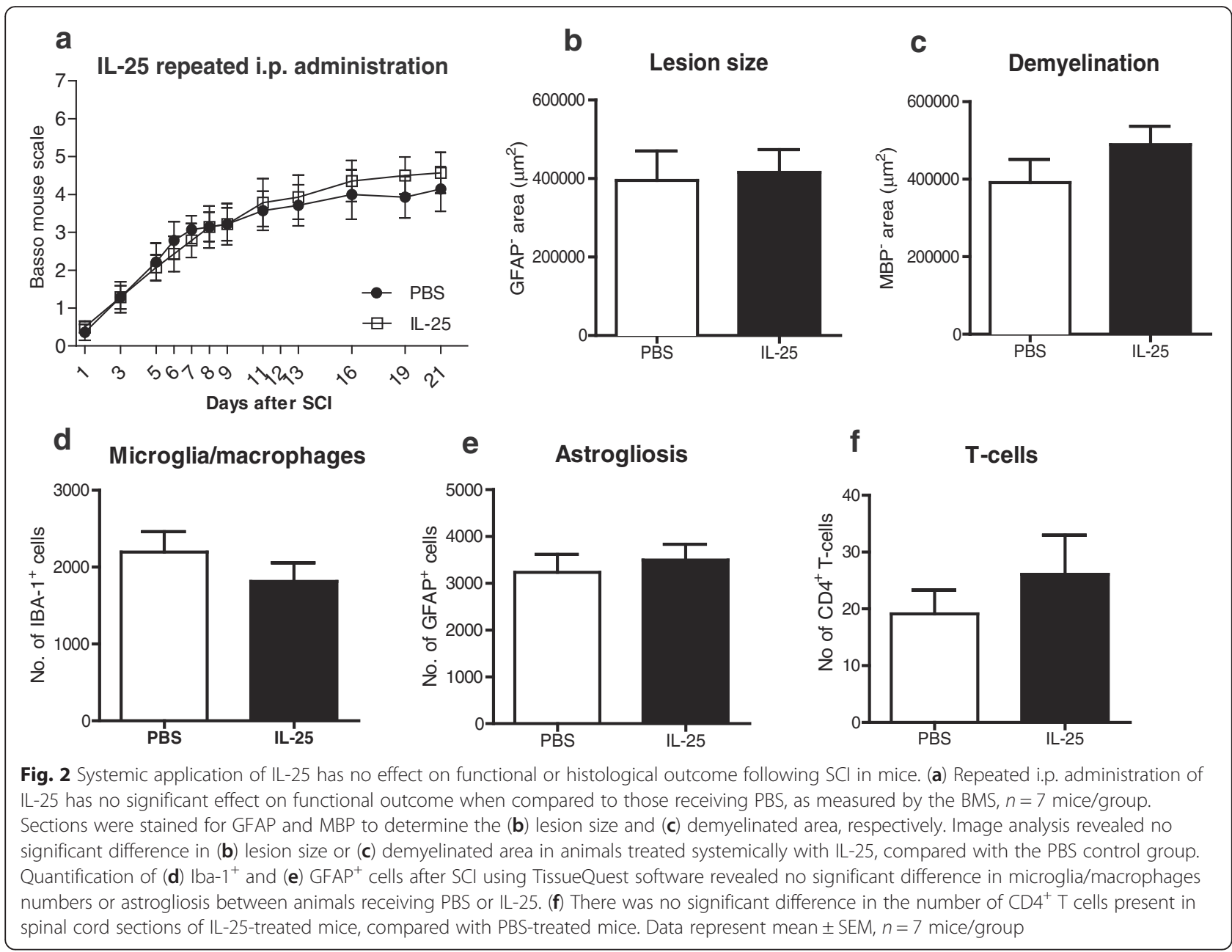

In contrast to the above positive effects on neuroinflammation, our results indicate that systemic administration of IL-25 after SCI in mice is ineffective in improving functional outcome. This result was surprising given that we as well as others have shown that treatment with cytokines which induce a type 2 response, such as IL-4 and IL-10, are neuroprotective following SCI $[11,12]$. Differences in systemic versus local administration is a well-known phenomenon [30-32], and our results are consistent with this as local application of IL-25 decreased functional recovery after SCI. Furthermore, we observed that a lower local dose of IL$25(500 \mathrm{ng} / \mathrm{ml})$ leads to a non-significant trend towards a decrease in functional outcome after SCI (data not shown), indicating that route of administration and dosing are important factors which must be considered prior to use of cytokine therapy.

Interestingly, it was previously demonstrated that intraspinal treatment with IL-10 exacerbated damage and lesion size, while when given systemically, it improved recovery after SCI [12]. Taken together, these data reiterate the well-recognized fact that the route of administration is of pivotal importance when determining a therapeutic outcome. Additionally, Mearns et al. recently questioned the role of IL-25 in Th2 cell differentiation and the induction of potentially beneficial Th2-cell responses [33]. In contrast to previous reports, the authors used reporter mouse technology to show that IL-25 is dispensable during differentiation and development of Th2 cells [33]. In our study, IL-25 failed to have an effect systemically and even worsened functional outcome when applied locally. This suggests that the direct involvement of IL-25 in driving a Th2 response remains questionable. Furthermore, based on the current opinion on the role of Th2 cytokines following CNS injury [8], it is safe to suggest that factors which regulate the type 2 immune response are, in turn, key players in CNS pathology.

In this short report, we show for the first time that IL25 is either ineffective when applied systemically or detrimental to spinal cord recovery when applied locally. These findings indicate that the potential positive effects of IL-25 and its involvement in driving a beneficial type 2 immune response need to be carefully reconsidered prior to its use therapeutically. 


\section{Additional files}

Additional file 1: Supplementary Materials. Detailed description of materials and methods used throghout the manuscript, provided as supplementary information. (DOCX $35 \mathrm{~kb}$ )

Additional file 2: Figure S1. IL-25 has no effect on mature oligodendrocyte, astrocyte, microglia, or primary neuron cell viability. (A) MO3.13 cells were differentiated to mature oligodendrocytes using PMA for $72 \mathrm{~h}$ and were treated for $48 \mathrm{~h}$ with selected concentrations of IL-25. (B, C) The astrocytic and microglial cell lines (CCF and BV2, respectively) were treated for $48 \mathrm{~h}$ with selected concentrations of IL-25. (D) Primary neurons were incubated with selected concentrations of IL-25 for $48 \mathrm{~h}$ in the presence or absence of B27. B27 deprivation induced a decreased cell viability, but IL-25 treatment had no effect on this. The selected concentrations of IL-25 used for all cell types were $5 \mathrm{ng} / \mathrm{ml}, 50 \mathrm{ng} / \mathrm{ml}$, $500 \mathrm{ng} / \mathrm{ml}$, and $1 \mu \mathrm{g} / \mathrm{ml}$. Cell survival was measured using an MTT assay, and values are expressed as percentage of the control. (A-D) There was no significant effect observed on cell viability in all cell types tested. Data represent mean \pm SEM of one representative experiment (from two to three independent experiments) ${ }^{* * *} p<0.001$. (PDF $316 \mathrm{~kb}$ )

\section{Abbreviations}

BMS: Basso mouse scale; CNS: central nervous system; GFAP: glial fibrillary acidic protein; i.p.: intraperitoneal; Iba-1: ionized calcium-binding adaptor molecule 1; IL: interleukin; MBP: myelin basic protein; PBS: phosphatebuffered saline; $\mathrm{SCl}$ : spinal cord injury.

\section{Competing interests}

The authors declare that they have no competing interests.

\section{Authors' contributions}

DD participated in the study design, surgical procedures, collection and/or assembly of data, data analysis/interpretation, and manuscript writing. EL participated in the study design, data analysis/interpretation, and manuscript writing. PP participated in the data analysis/interpretation and manuscript writing. SH participated in the study conception and coordination and helped to draft the final manuscript. All authors read and approved the final manuscript.

\section{Acknowledgements}

The authors would like to acknowledge Dr. Leen Timmerman's for her technical assistance. This study was supported by grants from the Research Foundation Flanders (FWO Vlaanderen) to EL (1.2.703.10N, 1.2.703.13N) and to SH (G.0834.11N, G.0389.12, G.0A5813, G.0A14.13)

\section{Author details}

${ }^{1}$ Department of Morphology, Biomedical Research Institute, Hasselt University, Diepenbeek, Belgium. ${ }^{2}$ Laboratory of Experimental Hematology, Vaccine and Infectious Disease Institute (Vaxinfectio), University of Antwerp, Antwerp, Belgium

\section{Received: 10 December 2015 Accepted: 28 April 2016} Published online: 06 May 2016

\section{References}

1. David S, Lopez-Vales R, Wee YV. Harmful and beneficial effects of inflammation after spinal cord injury: potential therapeutic implications. Handb Clin Neurol. 2012;109:485-502. doi:10.1016/B978-0-444-52137-8. 00030-9.

2. Oliphant CJ, Barlow JL, McKenzie AN. Insights into the initiation of type 2 immune responses. Immunology. 2011;134(4):378-85. doi:10.1111/j.13652567.2011.03499.x

3. Vidal PM, Lemmens E, Avila A, Vangansewinkel T, Chalaris A, Rose-John S, et al. ADAM17 is a survival factor for microglial cells in vitro and in vivo after spinal cord injury in mice. Cell Death Dis. 2013;4(12):e954. doi:10.1038/cddis.2013.466.

4. Spellberg B, Edwards JE. Type 1/type 2 immunity in infectious diseases. Clin Infect Dis. 2001:32(1):76-102. doi:10.1086/317537.

5. Oswald IP, Gazzinelli RT, Sher A, James SL. IL-10 synergizes with IL-4 and transforming growth factor-beta to inhibit macrophage cytotoxic activity. J Immunol. 1992;148(11):3578-82.
6. de Waal MR, Figdor CG, Huijbens R, Mohan-Peterson S, Bennett B, Culpepper J, et al. Effects of IL-13 on phenotype, cytokine production, and cytotoxic function of human monocytes. Comparison with IL-4 and modulation by IFN-gamma or IL-10. J Immunol. 1993;151(11):6370-81.

7. Hendrix S, Nitsch R. The role of T helper cells in neuroprotection and regeneration. J Neuroimmunol. 2007;184(1-2):100-12. doi:10.1016/j.jneuroim. 2006.11.019.

8. Vidal PM, Lemmens E, Dooley D, Hendrix S. The role of "anti-inflammatory" cytokines in axon regeneration. Cytokine Growth Factor Rev. 2013:24(1):1-12. doi:10.1016/j.cytogfr.2012.08.008.

9. Golz G, Uhlmann L, Ludecke D, Markgraf N, Nitsch R, Hendrix S. The cytokine/neurotrophin axis in peripheral axon outgrowth. Eur J Neurosci. 2006;24(10):2721-30. doi:10.1111/j.1460-9568.2006.05155.x.

10. Koeberle PD, Gauldie J, Ball AK. Effects of adenoviral-mediated gene transfer of interleukin-10, interleukin-4, and transforming growth factor-beta on the survival of axotomized retinal ganglion cells. Neuroscience. 2004;125(4):90320. doi:10.1016/S0306-4522(03)00398-1.

11. Walsh JT, Hendrix S, Boato F, Smirnov I, Zheng J, Lukens JR, et al. MHCIIindependent CD4+ T cells protect injured CNS neurons via IL-4. J Clin Invest. 2015;125(2):699-714. doi:10.1172/JCI76210.

12. Brewer $\mathrm{KL}$, Bethea JR, Yezierski RP. Neuroprotective effects of interleukin-10 following excitotoxic spinal cord injury. Exp Neurol. 1999;159(2):484-93. doi:10.1006/exnr.1999.7173.

13. Zhou Z, Peng X, Insolera R, Fink DJ, Mata M. IL-10 promotes neuronal survival following spinal cord injury. Exp Neurol. 2009;220(1):183-90. doi:10.1016/j.expneurol.2009.08.018.

14. Fort MM, Cheung J, Yen D, Li J, Zurawski SM, Lo S, et al. IL-25 Induces IL-4, IL-5, and IL-13 and Th2-associated pathologies in vivo. Immunity. 2001;15(6): 985-95. doi:10.1016/s1074-7613(01)00243-6.

15. Monteleone G, Pallone F, Macdonald TT. Interleukin-25: a two-edged sword in the control of immune-inflammatory responses. Cytokine Growth Factor Rev. 2010;21(6):471-5. doi:10.1016/j.cytogfr.2010.05.001.

16. Saadoun D, Terrier B, Cacoub P. Interleukin-25: key regulator of inflammatory and autoimmune diseases. Curr Pharm Des. 2011;17(34):3781-5.

17. Sonobe $Y$, Takeuchi H, Kataoka K, Li H, Jin S, Mimuro M, et al. Interleukin-25 expressed by brain capillary endothelial cells maintains blood-brain barrier function in a protein kinase Cepsilon-dependent manner. J Biol Chem. 2009; 284(46):31834-42. doi:10.1074/jbc.M109.025940.

18. Kleinschek MA, Owyang AM, Joyce-Shaikh B, Langrish CL, Chen Y, Gorman DM, et al. IL-25 regulates Th17 function in autoimmune inflammation. J Exp Med. 2007;204(1):161-70. doi:10.1084/jem.20061738.

19. Boato F, Hendrix S, Huelsenbeck SC, Hofmann F, Grosse G, Djalali S, et al. C3 peptide enhances recovery from spinal cord injury by improved regenerative growth of descending fiber tracts. J Cell Sci. 2010;123(Pt 10): 1652-62. doi:10.1242/jcs.066050.

20. Nelissen S, Vangansewinkel T, Geurts N, Geboes L, Lemmens E, Vidal PM, et al. Mast cells protect from post-traumatic spinal cord damage in mice by degrading inflammation-associated cytokines via mouse mast cell protease 4. Neurobiol Dis. 2014;62:260-72. doi:10.1016/j.nbd.2013.09.012.

21. Tuszynski MH, Steward O. Concepts and methods for the study of axonal regeneration in the CNS. Neuron. 2012;74(5):777-91. doi:10.1016/j.neuron. 2012.05.006

22. Geurts N, Vangansewinkel T, Lemmens S, Nelissen S, Geboes L, Schwartz C et al. Basophils are dispensable for the recovery of gross locomotion after spinal cord hemisection injury. J Leukoc Biol. 2015. doi:10.1189/jlb.3AB0815-370R

23. Basso DM, Fisher LC, Anderson AJ, Jakeman LB, McTigue DM, Popovich PG. Basso mouse scale for locomotion detects differences in recovery after spinal cord injury in five common mouse strains. J Neurotrauma. 2006;23(5): 635-59. doi:10.1089/neu.2006.23.635.

24. Le Blon D, Hoornaert C, Daans J, Santermans E, Hens N, Goossens H, et al. Distinct spatial distribution of microglia and macrophages following mesenchymal stem cell implantation in mouse brain. Immunol Cell Biol. 2014;92(8):650-8. doi:10.1038/icb.2014.49.

25. Hashimura T, Tubbs RR, Connelly R, Caulfield MJ, Trindade CS, McMahon JT, et al. Characterization of two cell lines with distinct phenotypes and genotypes established from a patient with renal cell carcinoma. Cancer Res. 1989;49(24 Part 1):7064-71

26. Buntinx M, Moreels M, Vandenabeele F, Lambrichts I, Raus J, Steels $\mathrm{P}$, et al. Cytokine-induced cell death in human oligodendroglial cell lines: I. Synergistic effects of IFN- $\gamma$ and TNF-a on apoptosis. J Neurosci Res. 2004; 76(6):834-45. doi:10.1002/jnr.20118. 
27. Blasi E, Barluzzi R, Bocchini V, Mazzolla R, Bistoni F. Immortalization of murine microglial cells by a v-raf/v-myc carrying retrovirus. J Neuroimmunol. 1990;27(2):229-37. doi:10.1016/0165-5728(90)90073-V.

28. Gu C, Wu L, Li X. IL-17 family: cytokines, receptors and signaling. Cytokine. 2013;64(2):477-85. doi:10.1016/j.cyto.2013.07.022.

29. Maiorino C, Khorooshi R, Ruffini F, Lobner M, Bergami A, Garzetti L, et al. Lentiviral-mediated administration of IL-25 in the CNS induces alternative activation of microglia. Gene Ther. 2013;20(5):487-96. doi:10.1038/gt.2012.58.

30. Collins JM. Pharmacologic rationale for regional drug delivery. J Clin Oncol. 1984;2(5):498-504.

31. Kerzerho J, Wunsch D, Szely N, Meyer H-A, Lurz L, Röse L, et al. Effects of systemic versus local administration of corticosteroids on mucosal tolerance. J Immunol. 2012;188(1):470-6. doi:10.4049/jimmunol.1101405.

32. Boato F, Rosenberger $K$, Nelissen $S$, Geboes L, Peters EM, Nitsch R, et al. Absence of IL-1 13 positively affects neurological outcome, lesion development and axonal plasticity after spinal cord injury. J Neuroinflammation. 2013;10:6. doi:10.1186/1742-2094-10-6.

33. Mearns H, Forbes-Blom EE, Camberis M, Tang S-C, Kyle R, Harvie M, et al. IL25 exhibits disparate roles during Th2-cell differentiation versus effector function. Eur J Immunol. 2014;44(7):1976-80. doi:10.1002/eji.201344400.

Submit your next manuscript to BioMed Central and we will help you at every step:

- We accept pre-submission inquiries

- Our selector tool helps you to find the most relevant journal

- We provide round the clock customer support

- Convenient online submission

- Thorough peer review

- Inclusion in PubMed and all major indexing services

- Maximum visibility for your research

Submit your manuscript at www.biomedcentral.com/submit
Biomed Central 